\title{
Indestructibility under Adding Cohen Subsets and Level by Level Equivalence ${ }^{* \dagger}$
}

\author{
Arthur W. Apter ${ }^{\ddagger}$ \\ Department of Mathematics \\ Baruch College of CUNY \\ New York, New York 10010 USA \\ and \\ The CUNY Graduate Center, Mathematics \\ 365 Fifth Avenue \\ New York, New York 10016 USA \\ http://faculty.baruch.cuny.edu/apter \\ awapter@alum.mit.edu \\ January 7, 2008 \\ (revised May 30, 2008 and July 20, 2008)
}

\begin{abstract}
We construct a model for the level by level equivalence between strong compactness and supercompactness in which the least supercompact cardinal $\kappa$ has its strong compactness indestructible under adding arbitrarily many Cohen subsets. There are no restrictions on the large cardinal structure of our model.
\end{abstract}

\section{Introduction and Preliminaries}

Since the notion of level by level equivalence between strong compactness and supercompactness is central to this paper, we begin with its definition. Suppose $V$ is a model of ZFC in which for all regular cardinals $\kappa<\lambda, \kappa$ is $\lambda$ strongly compact iff $\kappa$ is $\lambda$ supercompact, except possibly if $\kappa$

*2000 Mathematics Subject Classifications: 03E35, 03E55.

${ }^{\dagger}$ Keywords: Supercompact cardinal, strongly compact cardinal, strong cardinal, indestructibility, Gitik iteration of Prikry-like forcings, level by level equivalence between strong compactness and supercompactness.

¥The author’s research was partially supported by PSC-CUNY grants and CUNY Collaborative Incentive grants. In addition, the author wishes to thank the two referees for many helpful comments, suggestions, and corrections which have been incorporated into the current version of the paper. 
is a measurable limit of cardinals $\delta$ which are $\lambda$ supercompact. Such a universe will be said to witness level by level equivalence between strong compactness and supercompactness. The exception is provided by a theorem of Menas [21], who showed that if $\lambda \geq \kappa$ and $\kappa$ is a measurable limit of cardinals $\delta$ each of which is $\lambda$ strongly compact, then $\kappa$ is $\lambda$ strongly compact, but need not be $\lambda$ supercompact. We will therefore say that $\kappa$ is a witness to a failure of level by level equivalence between strong compactness and supercompactness iff there is a regular $\lambda>\kappa$ such that $\kappa$ is $\lambda$ strongly compact but is not a measurable limit of cardinals $\delta$ which are $\lambda$ supercompact, and $\kappa$ is not $\lambda$ supercompact. Note that inductively, $\kappa$ witnesses a failure of level by level equivalence between strong compactness and supercompactness iff $\lambda>\kappa$ is regular, $\kappa$ is $\lambda$ strongly compact but is not a measurable limit of cardinals $\delta$ which are $\lambda$ strongly compact, and $\kappa$ is not $\lambda$ supercompact. Models in which level by level equivalence between strong compactness and supercompactness holds nontrivially were first constructed in [12].

In [7], the following question was posed: Is it possible to construct a model for the level by level equivalence between strong compactness and supercompactness in which the least supercompact cardinal $\kappa$ has its supercompactness indestructible under partial orderings of the form $\operatorname{Add}(\kappa, \lambda)$, where $\lambda$ is some ordinal? ${ }^{1}$ Note that earlier indestructibility theorems for the least supercompact cardinal in conjunction with level by level equivalence between strong compactness and supercompactness may be found in the papers [11], [1], [7], and [5].

The purpose of this paper is to establish a result which provides a partial answer to the aforementioned question. Specifically, we prove the following theorem.

Theorem 1 Suppose $V \vDash " Z F C+\kappa$ is the least supercompact cardinal". There is then a partial ordering $\mathbb{P} \subseteq V$ such that $V^{\mathbb{P}} \vDash " Z F C+\kappa$ is the least supercompact cardinal + Level by level equivalence between strong compactness and supercompactness holds". In $V^{\mathbb{P}}, \kappa^{\prime}$ s strong compactness is indestructible under partial orderings of the form $\operatorname{Add}(\kappa, \lambda)$, where $\lambda$ is an arbitrary ordinal.

We take this opportunity to note that [5, Theorem 1] gives the existence of a model containing a supercompact cardinal $\kappa$ in which both level by level equivalence between strong compactness and

\footnotetext{
${ }^{1}$ For $\delta$ a regular cardinal and $\gamma$ an ordinal, $\operatorname{Add}(\delta, \gamma)$ is the standard partial ordering for adding $\gamma$ many Cohen subsets of $\delta$. To avoid trivialities, we always assume that $\gamma \geq 1$.
} 
supercompactness holds and $\kappa$ 's strong compactness is indestructible under arbitrary $\kappa$-directed closed forcing. However, in this model, the large cardinal structure is severely restricted, as no cardinal is supercompact up to an inaccessible cardinal. In particular, $\kappa$ is the only supercompact cardinal, and there are no inaccessible cardinals above $\kappa$. (As mentioned in [5], a modification of the forcing presented allows for a witnessing model which can contain inaccessibles above $\kappa$, but no measurables. Whether a further modification is possible allowing measurables above $\kappa$ is unknown.) In Theorem 1 of this paper, although we have far less indestructibility (and therefore prima facie a seemingly weaker result), there are no restrictions placed on the large cardinal structure of our witnessing model, which can contain supercompact limits of supercompact cardinals, etc. Thus, because of our methods of proof, in the presence of level by level equivalence between strong compactness and supercompactness, we have a tradeoff. On the one hand, it is possible to have full indestructibility for strong compactness for the least supercompact cardinal and a rather impoverished large cardinal structure. On the other hand, it is possible to have limited indestructibility for strong compactness for the least supercompact cardinal and a rich large cardinal structure.

We now very briefly give some preliminary information concerning notation and terminology. When forcing, $q \geq p$ means that $q$ is stronger than $p$, and $p \| \varphi$ means that $p$ decides $\varphi$. For $\alpha<\beta$ ordinals, $[\alpha, \beta]$ and $(\alpha, \beta]$ are as in standard interval notation. If $G$ is $V$-generic over $\mathbb{P}$, we will abuse notation slightly and use both $V[G]$ and $V^{\mathbb{P}}$ to indicate the universe obtained by forcing with $\mathbb{P}$. We will, from time to time, confuse terms with the sets they denote and write $x$ when we actually mean $\dot{x}$ or $\check{x}$.

The partial ordering $\mathbb{P}$ is $\kappa$-directed closed if every directed set of conditions of size less than $\kappa$ has an upper bound. $\mathbb{P}$ is $\kappa$-strategically closed if in the two person game in which the players construct an increasing sequence $\left\langle p_{\alpha}: \alpha \leq \kappa\right\rangle$, where player I plays odd stages and player II plays even stages (choosing the trivial condition at stage 0), player II has a strategy which ensures the game can always be continued. Note that if $\mathbb{P}$ is $\kappa$-strategically closed and $f: \kappa \rightarrow V$ is a function in $V^{\mathbb{P}}$, then $f \in V$.

As in [16], if $\mathcal{A}$ is a collection of partial orderings, then the lottery sum is the partial ordering 
$\oplus \mathcal{A}=\{\langle\mathbb{P}, p\rangle: \mathbb{P} \in \mathcal{A}$ and $p \in \mathbb{P}\} \cup\{0\}$, ordered with 0 below everything and $\langle\mathbb{P}, p\rangle \leq\left\langle\mathbb{P}^{\prime}, p^{\prime}\right\rangle$ iff $\mathbb{P}=\mathbb{P}^{\prime}$ and $p \leq p^{\prime}$. Intuitively, if $G$ is $V$-generic over $\oplus \mathcal{A}$, then $G$ first selects an element of $\mathcal{A}$ (or as Hamkins says in [16], "holds a lottery among the posets in $\mathcal{A}$ ") and then forces with it. The terminology "lottery sum" is due to Hamkins, although the concept of the lottery sum of partial orderings has been around for quite some time and has been referred to at different junctures via the expressions "disjoint sum of partial orderings," "side-by-side forcing," and "choosing which partial ordering to force with generically."

The partial ordering $\mathbb{P}$ which will be used in the proof of Theorem 1 is a Gitik iteration of Prikry-like forcings. As such, we take this opportunity to mention some of the basic properties of this sort of iteration.

We begin our discussion with a preliminary definition found in [14]. Suppose $\delta$ is inaccessible. A partial ordering $\mathbb{Q}$ is $\delta$-weakly closed and satisfies the Prikry property if it meets the following criteria.

1. $\mathbb{Q}$ has two partial orderings $\leq$ and $\leq^{*}$, with $\leq^{*} \subseteq \leq$.

2. For any condition $p \in \mathbb{Q}$ and any formula $\varphi$ in the forcing language with respect to $\mathbb{Q}$, there is some $q \geq^{*} p$ such that $q \| \varphi$.

3. For each $\gamma<\delta$, any $\leq^{*}$ increasing chain of elements of $\mathbb{Q}$ of length $\gamma$ has an upper bound.

Given the above definition, a Gitik iteration $\mathbb{P}$ of Prikry-like forcings having length $\kappa$ is an Easton support iteration $\left\langle\left\langle\mathbb{P}_{\alpha}, \dot{\mathbb{Q}}_{\alpha}\right\rangle: \alpha<\kappa\right\rangle$ in which at each nontrivial stage $\delta$ (which for our purposes will always be inaccessible), the forcing used is $\delta$-weakly closed and satisfies the Prikry property. In our situation, each component $\dot{\mathbb{Q}}_{\delta}$ of the iteration used at a nontrivial stage $\delta$ has the form $\dot{\mathbb{Q}}_{\delta}^{0} * \dot{\mathbb{Q}}_{\delta}^{1} * \dot{\mathbb{Q}}_{\delta}^{2}$, where $\dot{\mathbb{Q}}_{\delta}^{0}$ is a term for a certain $\delta$-directed closed partial ordering, $\dot{\mathbb{Q}}_{\delta}^{1}$ is a term for either trivial forcing or Prikry forcing, and $\dot{\mathbb{Q}}_{\delta}^{2}$ is a term for a certain $\delta$-strategically closed partial ordering. (That a $\delta$-strategically closed partial ordering may be used as a component in a Gitik iteration of Prikry-like forcings was shown in [6].) 
The ordering $\leq$ on $\mathbb{P}$ is the standard ordering used when an Easton support iteration is defined except, roughly speaking, the stems of Prikry conditions are extended nontrivially only finitely often. Its definition is given in [13], [14], and [10], but for concreteness, we repeat it here. Specifically, let $p, q \in \mathbb{P}, p=\left\langle\dot{p}_{\alpha}: \alpha<\kappa\right\rangle, q=\left\langle\dot{q}_{\alpha}: \alpha<\kappa\right\rangle$. Then $q \geq p$ iff $q$ extends $p$ with respect to the usual Easton support iteration ordering, but in addition, for some finite $A \subseteq \operatorname{support}(p)$ and all

$\beta \in \operatorname{support}(p)-A$, for $\dot{q}_{\beta}=\left\langle\dot{r}_{\beta}^{\prime}, \dot{s}_{\beta}^{\prime}, \dot{t}_{\beta}^{\prime}\right\rangle, \dot{p}_{\beta}=\left\langle\dot{r}_{\beta}, \dot{s}_{\beta}, \dot{t}_{\beta}\right\rangle, q \uparrow \beta * \dot{r}_{\beta}^{\prime} \in \mathbb{P}_{\beta} * \dot{\mathbb{Q}}_{\beta}^{0}, p \uparrow \beta * \dot{r}_{\beta} \in \mathbb{P}_{\beta} * \dot{\mathbb{Q}}_{\beta}^{0}$,

$q \uparrow \beta * \dot{r}_{\beta}^{\prime} \Vdash_{\mathbb{P}_{\beta} * \dot{\mathbb{Q}}_{\beta}^{0}}$ "If $\dot{s}_{\beta}^{\prime}$ and $\dot{s}_{\beta}$ are conditions with respect to Prikry forcing, then $\dot{s}_{\beta}^{\prime}$ and $\dot{s}_{\beta}$ have the same stems". (Intuitively, this means that $s_{\beta}^{\prime}$ is obtained from $s_{\beta}$ "by shrinking measure 1 sets".) Further, if $A=\emptyset$ in the above definition, then $q$ is called an Easton extension of $p$.

By [13, Lemma 1.4], for any $p \in \mathbb{P}$ and any formula $\varphi$ in the language of forcing with respect to $\mathbb{P}$, there is some Easton extension $q$ of $p$ such that $q \| \varphi$. By [13, Lemma 1.2], if $\delta$ is the least cardinal acted upon nontrivially and for some $\gamma<\delta,\left\langle p_{\alpha}: \alpha<\gamma\right\rangle$ is a sequence of elements of $\mathbb{P}$ such that $\alpha<\beta$ implies $p_{\beta}$ is an Easton extension of $p_{\alpha}$, then there is some condition $p \in \mathbb{P}$ which is an Easton extension of each $p_{\alpha}$. Therefore, in analogy to Prikry forcing, forcing with $\mathbb{P}$ adds no new bounded subsets of $\delta$. Thus, [13, Lemmas 1.2 and 1.4] show that $\mathbb{P}$ is $\delta$-weakly closed and satisfies the Prikry property, with $\leq^{*}$ defined as Easton extension.

We assume familiarity with the large cardinal notions of measurability, strongness, strong compactness, and supercompactness. Readers are urged to consult [17] for further details. We do wish to point out explicitly, however, that an indestructibly supercompact cardinal $\kappa$ is one as in [19], i.e., a supercompact cardinal which remains supercompact after $\kappa$-directed closed forcing. We say that $\kappa$ is supercompact (or strongly compact) up to the cardinal $\lambda$ if $\kappa$ is $\gamma$ supercompact (or $\gamma$ strongly compact) for every $\gamma<\lambda$. Finally, for any ordinal $\delta, \delta^{\prime}$ will denote the least strong cardinal in the ground model $V$ greater than $\delta$.

\section{The Proof of Theorem 1}

We turn now to the proof of Theorem 1.

Proof: Let $V \vDash$ "ZFC $+\kappa$ is the least supercompact cardinal". By first forcing GCH and 
then forcing as in [12], we also assume in addition that $V \vDash$ "GCH + Level by level equivalence between strong compactness and supercompactness holds". The goal now is to preserve level by level equivalence between strong compactness and supercompactness and the fact $\kappa$ is the least supercompact cardinal, while forcing indestructibility of the strong compactness of $\kappa$ under partial orderings of the form $\operatorname{Add}(\kappa, \lambda)$, where $\lambda$ is an arbitrary ordinal.

The partial ordering $\mathbb{P}$ used in the proof of Theorem 1 is a length $\kappa$ Gitik iteration of Prikry-like forcings $\left\langle\left\langle\mathbb{P}_{\alpha}, \dot{\mathbb{Q}}_{\alpha}\right\rangle: \alpha<\kappa\right\rangle$. Specifically, $\mathbb{P}_{0}$ is trivial forcing $\{\emptyset\}$. The only nontrivial stages of forcing $\delta<\kappa$ occur at cardinals which are measurable limits of strong cardinals in $V$. At such a stage $\delta, \dot{\mathbb{Q}}_{\delta}$ has the form $\dot{\mathbb{Q}}_{\delta}^{0} * \dot{\mathbb{Q}}_{\delta}^{1} * \dot{\mathbb{Q}}_{\delta}^{2}$, where $\dot{\mathbb{Q}}_{\delta}^{0}$ is a term for the lottery sum of both trivial forcing and all partial orderings of the form $\operatorname{Add}(\delta, \lambda)$ having rank below $\delta^{\prime}$. If $\vdash_{\mathbb{P}_{\delta} * \dot{\mathbb{Q}}_{\delta}^{0}}$ "Level by level equivalence between strong compactness and supercompactness fails at $\delta$ ", then $\dot{\mathbb{Q}}_{\delta}^{1}$ is a term for Prikry forcing over $\delta$ defined with respect to some normal measure; otherwise, $\dot{\mathbb{Q}}_{\delta}^{1}$ is a term for trivial forcing. No matter what $\dot{\mathbb{Q}}_{\delta}^{1}$ is, $\dot{\mathbb{Q}}_{\delta}^{2}$ is a term for the partial ordering adding a non-reflecting stationary set of ordinals of cofinality $\omega$ to $\delta^{\prime}$. (Note that a precise definition of the partial ordering for adding a non-reflecting stationary set of ordinals of cofinality $\omega$ to a limit cardinal $\gamma$ may be found in, e.g., [8, Section 1, page 1898]. A property of this partial ordering which we use here is that it is $\rho$-strategically closed for every $\rho<\gamma$.)

Lemma 2.1 Suppose $\delta \leq \kappa$ is a measurable limit of strong cardinals in $V$. Then $\Vdash_{\mathbb{P}_{\delta}}$ "Level by level equivalence between strong compactness and supercompactness holds at $\delta$ ".

Proof: We use ideas from the proof of [5, Lemma 2.1]. Since $V \vDash$ GCH, by [13, Lemma 1.5], $\Vdash_{\mathbb{P}_{\delta}}$ " $\delta$ is a measurable cardinal". We consequently assume inductively that for every cardinal $\gamma<\delta$ which is a measurable limit of strong cardinals in $V, \Vdash_{\mathbb{P}_{\gamma}}$ "Level by level equivalence between strong compactness and supercompactness holds at $\gamma^{\prime \prime}$.

Let $\lambda>\delta$ be a regular cardinal in $V^{\mathbb{P}_{\delta}}$ such that $\vdash_{\mathbb{P}_{\delta}}$ " $\delta$ is $\lambda$ strongly compact". Note that because $\delta$ is measurable and hence inaccessible in $V, \mathbb{P}_{\delta}$ is the direct limit of $\left\langle\mathbb{P}_{\alpha}: \alpha<\delta\right\rangle$. In addition, $\mathbb{P}_{\delta}$ satisfies $\delta$-c.c. in $V^{\mathbb{P}_{\delta}}$, since $\delta$ is measurable and hence Mahlo in $V^{\mathbb{P}_{\delta}}$ and $\mathbb{P}_{\delta}$ is a subordering of the direct limit of $\left\langle\mathbb{P}_{\alpha}: \alpha<\delta\right\rangle$ as calculated in $V^{\mathbb{P}_{\delta}}$. Hence, by [15, Theorem 2.1.5] 
(see also the proofs of [8, Lemma 3] or [4, Lemma 8]), every $\delta$-additive uniform ultrafilter over a regular cardinal $\gamma \geq \delta$ present in $V^{\mathbb{P}_{\delta}}$ must be an extension of a $\delta$-additive uniform ultrafilter over $\gamma$ in $V$. Therefore, since the $\lambda$ strong compactness of $\delta$ in $V^{\mathbb{P}_{\delta}}$ implies that every $V^{\mathbb{P}_{\delta}}$-regular cardinal $\gamma \in[\delta, \lambda]$ carries a $\delta$-additive uniform ultrafilter in $V^{\mathbb{P}_{\delta}}$, and since the fact that $\mathbb{P}_{\delta}$ is $\delta$-c.c. tells us that the regular cardinals at or above $\delta$ in $V^{\mathbb{P}_{\delta}}$ are the same as those in $V$, the preceding sentence implies that every $V$-regular cardinal $\gamma \in[\delta, \lambda]$ carries a $\delta$-additive uniform ultrafilter in $V$. Ketonen's theorem of [18] then implies that $\delta$ is $\lambda$ strongly compact in $V$. By the fact $V \vDash$ "Level by level equivalence between strong compactness and supercompactness holds", $V \vDash$ "Either $\delta$ is $\lambda$ supercompact, or $\delta$ is a measurable limit of cardinals $\gamma$ which are $\lambda$ supercompact". If $V \vDash$ " $\delta$ is a measurable limit of cardinals $\gamma$ which are $\lambda$ supercompact", then because $\delta$ is a measurable limit of strong cardinals in $V$ and $\lambda \geq \delta$, for some $\gamma<\delta$ which is $\lambda$ supercompact in $V, V \vDash$ " $\gamma$ is supercompact up to $\gamma^{\prime \prime}$. By [3, Lemma 1.1], $V \vDash$ " $\gamma$ is supercompact" ${ }^{2}$, which contradicts that $V \vDash " \gamma<\kappa$ and $\kappa$ is the least supercompact cardinal". Thus, $V \vDash$ " $\delta$ is $\lambda$ supercompact". The proof of Lemma 2.1 will therefore be complete once we have shown that $\vdash_{\mathbb{P}_{\delta}}$ " $\delta$ is $\lambda$ supercompact".

To do this, fix $j: V \rightarrow M$ an elementary embedding witnessing the $\lambda$ supercompactness of $\delta$. By choosing $j(\delta)$ to be minimal, we may assume in addition that $M \vDash$ " $\delta$ is not $\lambda$ supercompact". Because $\lambda>\delta$ and $\operatorname{cp}(j)=\delta$, by GCH in $V, M \vDash " \delta$ is a measurable limit of strong cardinals". Therefore, since $j(\delta)>\lambda>\delta$, our inductive assumptions in $V$ imply that in $M, \Vdash_{\mathbb{P}_{\delta}}$ " $\delta$ is a measurable cardinal and level by level equivalence between strong compactness and supercompactness holds at $\delta$ ". Also, at stage $\delta$ in $M$ in the definition of $j\left(\mathbb{P}_{\delta}\right), \dot{\mathbb{Q}}_{\delta}=\left(\dot{\mathbb{Q}}_{\delta}^{0}\right)^{M} *\left(\dot{\mathbb{Q}}_{\delta}^{1}\right)^{M} *\left(\dot{\mathbb{Q}}_{\delta}^{2}\right)^{M}$, where $\left(\dot{\mathbb{Q}}_{\delta}^{0}\right)^{M}$ is a term for the stage $\delta$ lottery sum performed in the definition of $j\left(\mathbb{P}_{\delta}\right),\left(\dot{\mathbb{Q}}_{\delta}^{1}\right)^{M}$ is a term for either trivial forcing or Prikry forcing, and $\left(\dot{\mathbb{Q}}_{\delta}^{2}\right)^{M}$ is a term for a partial ordering which adds a non-reflecting stationary set of ordinals to $\left(\delta^{\prime}\right)^{M}$. Thus, if we opt for trivial forcing in the stage $\delta$ lottery sum done in $M^{\mathbb{P}_{\delta}}$ in the definition of $j\left(\mathbb{P}_{\delta}\right)$, our inductive assumptions allow us to take as $\left(\dot{\mathbb{Q}}_{\delta}^{1}\right)^{M}$ a term for trivial forcing. Because $M \vDash " \delta$ is not $\lambda$ supercompact", again by [3, Lemma 1.1], $M \vDash$ "No cardinal $\gamma \in(\delta, \lambda]$ is strong". Hence, $\left(\delta^{\prime}\right)^{M}>\lambda$. Consequently, above the appropriate

\footnotetext{
${ }^{2}$ As one of the referees has pointed out, the fact that $V \vDash$ " $\gamma$ is supercompact" follows as well from the fact that because $\gamma^{\prime}$ is strong, $V_{\gamma^{\prime}}$ is $\Sigma_{2}$ correct. This use of $\Sigma_{2}$ correctness will also be applicable elsewhere in the paper.
} 
condition in $M, j\left(\mathbb{P}_{\delta}\right)$ is forcing equivalent to $\mathbb{P}_{\delta} * \dot{\mathbb{P}}^{*}$, where $\dot{\mathbb{P}}^{*}$ is a term for a Gitik iteration of Prikry-like forcings whose first nontrivial stage takes place at $\left(\delta^{\prime}\right)^{M}$, i.e., well beyond $\lambda$. Since $V \vDash \mathrm{GCH}$, we may now apply the argument found in [2, Lemma 2.1] (see also [13, Lemma 1.5]) to show that $\Vdash_{\mathbb{P}_{\delta}}$ " $\delta$ is $\lambda$ supercompact". This completes the proof of Lemma 2.1.

Lemma 2.2 If $\delta \leq \kappa$ is in $V$ a measurable limit of strong cardinals, $\lambda>\delta$ is regular, and $V \vDash " \delta$ is $\lambda$ supercompact", then $\Vdash_{\mathbb{P}_{\delta}}$ " $\delta$ is $\lambda$ supercompact".

Proof: We use ideas from the proof of $[5$, Lemma 2.2]. Since $V \vDash$ " $\delta$ is $\lambda$ supercompact", let $j: V \rightarrow M$ be an elementary embedding witnessing this fact such that $M \vDash$ " $\delta$ is not $\lambda$ supercompact". As in Lemma 2.1, $M \vDash$ " $\delta$ is a measurable limit of strong cardinals". Therefore, by Lemma 2.1 applied in $M, \Vdash_{\mathbb{P}_{\delta}}$ " $\delta$ is a measurable cardinal and level by level equivalence between strong compactness and supercompactness holds at $\delta "$. Consequently, the argument given in the last paragraph of the proof of Lemma 2.1 now applies to show that in $V$, $\Vdash_{\mathbb{P}_{\delta}}$ " $\delta$ is $\lambda$ supercompact". This completes the proof of Lemma 2.2.

Since in Lemma 2.2, $\lambda$ can be arbitrary, and since by [9, Lemma 2.1] and the succeeding remarks, any supercompact cardinal is a limit of strong cardinals, it immediately follows that in $V^{\mathbb{P}}, \kappa$ is supercompact.

Lemma 2.3 $V^{\mathbb{P}} \vDash$ "Level by level equivalence between strong compactness and supercompactness holds below $\kappa "$.

Proof: The first part of the proof of Lemma 2.3 uses ideas from the proof of [5, Lemma 2.4]. Suppose $\delta<\kappa$ is in $V$ a measurable limit of strong cardinals and $V^{\mathbb{P}} \vDash " \lambda>\delta$ is a regular cardinal and $\delta$ is $\lambda$ strongly compact". Since $\delta$ is a stage at which a nontrivial forcing takes place, we may write $\mathbb{P}=\left(\mathbb{P}_{\delta} * \dot{\mathbb{Q}}_{\delta}^{0} * \dot{\mathbb{Q}}_{\delta}^{1}\right) *\left(\dot{\mathbb{Q}}_{\delta}^{2} * \dot{\mathbb{S}}^{\prime}\right)=\mathbb{R} * \dot{\mathbb{S}}$. We will be working above a condition forcing 
the statement " $\lambda>\delta$ is a regular cardinal and $\delta$ is $\lambda$ strongly compact". With a slight abuse of notation, we assume this condition is the trivial one.

By [13, Lemmas 1.4 and 1.2] and the definition of $\mathbb{P}$ as a Gitik iteration of Prikry-like forcings, $\Vdash_{\mathbb{R}}$ "Forcing with $\dot{\mathbb{S}}$ does not add bounded subsets of $\delta^{\prime}$ but does add a non-reflecting stationary set of ordinals of cofinality $\omega$ to $\delta^{\prime \prime \prime}$. By [22, Theorem 4.8] and the succeeding remarks, if $\gamma$ is $\eta$ strongly compact and $\eta$ is regular, then $\eta$ must reflect stationary sets of ordinals of cofinality $\omega$. Consequently, $\Vdash_{\mathbb{P}}$ " $\delta$ is not $\delta^{\prime}$ strongly compact". Thus, by the preceding three sentences, $\Vdash_{\mathbb{P}}$ " $\lambda<\delta^{\prime \prime}$ ", and $\Vdash_{\mathbb{R}}$ " $\delta$ is $\lambda$ strongly compact". Therefore, since $\mathbb{R}=\mathbb{P}_{\delta} * \dot{\mathbb{Q}}_{\delta}^{0} * \dot{\mathbb{Q}}_{\delta}^{1}$ and $\dot{\mathbb{Q}}_{\delta}^{1}$ must be a term either for trivial forcing or for Prikry forcing over $\delta, \dot{\mathbb{Q}}_{\delta}^{1}$ is a term for trivial forcing, and $\Vdash_{\mathbb{P}_{\delta} * \dot{\mathbb{Q}}_{\delta}^{0}}$ " $\delta$ is $\lambda$ strongly compact". Hence, as $\dot{\mathbb{Q}}_{\delta}^{1}$ is not a term for Prikry forcing over $\delta$, by the definition of $\mathbb{P}, \Vdash_{\mathbb{P}_{\delta} * \dot{\mathbb{Q}}_{\delta}^{0}}$ "Either $\delta$ is $\lambda$ supercompact, or $\delta$ is a measurable limit of cardinals $\gamma$ which are $\lambda$ supercompact". The preceding then immediately implies that $\Vdash_{\mathbb{P}_{\delta} * \dot{\mathbb{Q}}_{\delta}^{0} * \dot{\mathbb{Q}}_{\delta}^{1} * \dot{\mathbb{S}}}$ "Either $\delta$ is $\lambda$ supercompact, or $\delta$ is a measurable limit of cardinals $\gamma$ which are $\lambda$ supercompact", i.e., $V^{\mathbb{P}} \vDash$ " $\delta$ is not a witness to a failure of level by level equivalence between strong compactness and supercompactness".

Suppose now that $\delta<\kappa$ is not in $V$ a measurable limit of strong cardinals, and that in addition, $V^{\mathbb{P}} \vDash " \lambda>\delta$ is a regular cardinal and $\delta$ is $\lambda$ strongly compact". As before, we will be working above a condition forcing the statement " $\lambda>\delta$ is a regular cardinal and $\delta$ is $\lambda$ strongly compact". Once again, with a slight abuse of notation, we assume this condition is the trivial one.

Let $A=\left\{\gamma \leq \delta: \gamma\right.$ is either a measurable limit of strong cardinals in $V$ or $\gamma=\eta^{\prime}$ for some $\eta$ which is in $V$ a measurable limit of strong cardinals\}. Define $\rho$ as the least ordinal greater than or equal to the supremum of $A$ such that for the factorization $\mathbb{P}=\mathbb{P}_{\rho} * \dot{\mathbb{Q}}, \Vdash_{\mathbb{P}_{\rho}}$ "For all $\gamma \in A$, forcing with $\dot{\mathbb{Q}}$ adds no new subsets of $\gamma$ ". First, note that $|A|<\delta$. To see this, assume not, i.e., suppose that $|A|=\delta$. We then have that $\delta \neq \eta^{\prime}$ for any $\eta<\delta$ which is in $V$ a measurable limit of strong cardinals. This is since $\delta=\eta^{\prime}$ for such an $\eta$ would immediately imply that $\delta$ were the largest member of $A$ and $|A|<\delta$. Because $|A|=\delta$ and $\delta$ is not in $V$ a measurable limit of strong cardinals, $\delta \notin A$. From this, it immediately follows that $\rho=\delta$. Since the definition of $\mathbb{P}$ then implies that 
$\Vdash_{\mathbb{P}_{\delta}}$ "Forcing with $\dot{\mathbb{Q}}$ adds no new subsets of $2^{\delta} ", V^{\mathbb{P}_{\delta}} \vDash " \delta$ is measurable". In addition, as forcing can't create new Mahlo cardinals and $V^{\mathbb{P}} \vDash$ " $\delta$ is measurable and hence Mahlo", $V \vDash$ " $\delta$ is a Mahlo limit of nontrivial stages of forcing". The argument given in the second paragraph of the proof of Lemma 2.1 therefore applies and shows that $V \vDash " \delta$ is measurable". Hence, $\delta$ is in $V$ a measurable limit of strong cardinals, a contradiction.

Because $|A|<\delta$, it must therefore be the case that $\mathbb{P}_{\rho}$ is forcing equivalent to a partial ordering having size less than $\delta$. This is since otherwise, by the definition of $\mathbb{P}$, there must be some $\gamma<\delta$ which is in $V$ the largest measurable limit of strong cardinals below $\delta$. It is true that $\delta \leq \gamma^{\prime}$ (if $\left.\delta>\gamma^{\prime},\left|\mathbb{P}_{\rho}\right|<\delta\right)$. We cannot then have that $\delta=\gamma^{\prime}$, since if so, $V^{\mathbb{P}} \vDash " \delta$ contains a non-reflecting stationary set of ordinals of cofinality $\omega$ and hence is non-weakly compact". As we must thus have that $\delta<\gamma^{\prime}$, the stage $\gamma$ lottery must select a partial ordering $\mathbb{Q}^{*}$ such that $\left|\mathbb{Q}^{*}\right| \geq \delta$. It then follows that $2^{\gamma} \geq \delta$ in both $V^{\mathbb{P} \rho}$ and $V^{\mathbb{P}_{\rho} * \dot{\mathbb{Q}}}=V^{\mathbb{P}}$, a contradiction to the fact that $V^{\mathbb{P}} \vDash$ " $\delta$ is measurable".

Now, as $\dot{\mathbb{Q}}$ has to be a term for a Gitik iteration of Prikry-like forcings whose first nontrivial stage is at or above $\delta^{\prime}$, again by $\left[13\right.$, Lemmas 1.4 and 1.2], it must be the case that $\vdash_{\mathbb{P}_{\rho}}$ "Forcing with $\dot{\mathbb{Q}}$ adds no new bounded subsets of $\delta^{\prime \prime}$. We must therefore have that $\lambda<\delta^{\prime}$, for if not, then it must be true that in both $V^{\mathbb{P}_{\rho}}$ and $V^{\mathbb{P}_{\rho} * \dot{\mathbb{Q}}}=V^{\mathbb{P}}, \delta$ is strongly compact up to $\delta^{\prime}$. Since $\mathbb{P}_{\rho}$ is forcing equivalent to a partial ordering having size less than $\delta$, by the Lévy-Solovay results [20], $V \vDash " \delta<\kappa$ is strongly compact up to $\delta^{\prime \prime}$. Hence, again by [3, Lemma 1.1], $V \vDash " \delta$ is strongly compact", a contradiction to the fact that by level by level equivalence between strong compactness and supercompactness in $V, V \vDash " \kappa$ is both the least strongly compact and least supercompact cardinal".

Since forcing with $\mathbb{Q}$ over $V^{\mathbb{P} \rho}$ adds no new bounded subsets of $\delta^{\prime}$, we may consequently conclude that $\delta$ is $\lambda$ strongly compact in both $V^{\mathbb{P}_{\rho}}$ and $V^{\mathbb{P}_{\rho} * \dot{\mathbb{Q}}}=V^{\mathbb{P}}$. Again by the results of $[20], V \vDash " \delta$ is $\lambda$ strongly compact" as well. Thus, by level by level equivalence between strong compactness and supercompactness in $V, V \vDash$ "Either $\delta$ is $\lambda$ supercompact, or $\delta$ is a measurable limit of cardinals $\gamma$ which are $\lambda$ supercompact". Once more using the results of [20] and the fact that forcing with $\mathbb{Q}$ over $V^{\mathbb{P} \rho}$ adds no new bounded subsets of $\delta^{\prime}$, in both $V^{\mathbb{P} \rho}$ and $V^{\mathbb{P}_{\rho} * \dot{\mathbb{Q}}}=V^{\mathbb{P}}$, either $\delta$ is $\lambda$ 
supercompact, or $\delta$ is a measurable limit of cardinals $\gamma$ which are $\lambda$ supercompact. In either case, $\delta$ is not a witness in $V^{\mathbb{P}}$ to a failure of level by level equivalence between strong compactness and supercompactness. As $\delta$ and $\lambda$ were arbitrary, this completes the proof of Lemma 2.3.

Because $\mathbb{P}$ may be defined so that $|\mathbb{P}|=\kappa$, by the results of $[20], V^{\mathbb{P}} \vDash$ "Level by level equivalence between strong compactness and supercompactness holds above $\kappa$ ". Since as we have already observed, $V^{\mathbb{P}} \vDash " \kappa$ is supercompact", Lemma 2.3 allows us to infer that $V^{\mathbb{P}} \vDash$ "Level by level equivalence between strong compactness and supercompactness holds". Also, note that it is only in the fifth paragraph of the proof of Lemma 2.3 that we use that nontrivial components of our lottery sums are forcings for adding Cohen subsets, rather than arbitrary $\gamma$-directed closed partial orderings.

Lemma 2.4 $V^{\mathbb{P}} \vDash$ “ $\kappa$ 's strong compactness is indestructible under forcing with partial orderings of the form $\operatorname{Add}(\kappa, \eta)$ for $\eta$ an arbitrary ordinal".

Proof: We use ideas from the proof of $\left[5\right.$, Lemma 2.6]. Suppose $\mathbb{Q} \in V^{\mathbb{P}}$ is such that $V^{\mathbb{P}} \vDash$

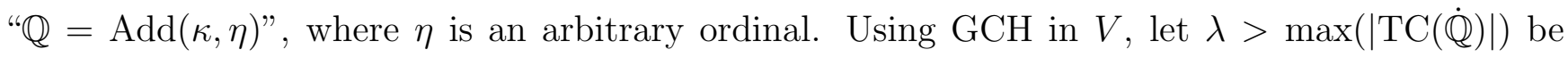
an arbitrary regular cardinal large enough so that $\left(2^{[\lambda]^{<\kappa}}\right)^{V}=\rho=\left(2^{[\lambda]^{<\kappa}}\right)^{V^{\mathbb{P} * \dot{\mathbb{Q}}}}$ and $\rho$ is regular in both $V$ and $V^{\mathbb{P} * \dot{\mathbb{Q}}}$, and let $\sigma=\rho^{+}=2^{\rho}=2^{[\rho]^{<\kappa}}$. Take as $j: V \rightarrow M$ an elementary embedding witnessing the $\sigma$ supercompactness of $\kappa$ such that $M \vDash$ " $\kappa$ is not $\sigma$ supercompact". Since as before, $M \vDash$ "No cardinal $\delta \in(\kappa, \sigma]$ is strong", $M \vDash$ " $|\mathrm{TC}(\dot{\mathbb{Q}})|$ is below the least strong cardinal above $\kappa "$. Thus, because as earlier, $M \vDash " \kappa$ is a measurable limit of strong cardinals", $\dot{\mathbb{Q}}$ is a term for an allowable choice in the stage $\kappa$ lottery sum performed in $M$ in the definition of $j(\mathbb{P})$. We hence for the rest of this proof force in $M$ above a condition $p^{*}$ that opts for $\mathbb{Q}$ in $M^{\mathbb{P}}$, so that above $p^{*}$, $j(\mathbb{P})$ is forcing equivalent to $\mathbb{P}_{\kappa} * \dot{\mathbb{Q}} * \dot{\mathbb{Q}}_{\kappa}^{1} *\left(\dot{\mathbb{Q}}_{\kappa}^{2} * \dot{\mathbb{R}}^{\prime}\right)=\mathbb{P} * \dot{\mathbb{Q}} * \dot{\mathbb{Q}}_{\kappa}^{1} * \dot{\mathbb{R}}$.

The remainder of the proof of Lemma 2.4, as with the proof of [5, Lemma 2.6], is as in the proof of [10, Lemma 2]. We therefore refer readers to the proof of [10, Lemma 2] for any missing details. By the last sentence of the preceding paragraph, in $M$, above $p^{*}, j(\mathbb{P} * \dot{\mathbb{Q}})$ is forcing equivalent 
to $\mathbb{P} * \dot{\mathbb{Q}} * \dot{\mathbb{Q}}_{\kappa}^{1} * \dot{\mathbb{R}} * j(\dot{\mathbb{Q}})$, where $\Vdash_{\mathbb{P} * \dot{\mathbb{Q}}}$ “这${ }_{\kappa}^{1}$ is a term for either Prikry forcing or trivial forcing". Further, since $M \vDash$ "No cardinal $\delta \in(\kappa, \sigma]$ is strong", the first nontrivial stage in the definition of $\dot{\mathbb{R}}$ after $\kappa$ takes place well above $\sigma$. Consequently, as in [10, Lemma 2], there is a term $\tau \in M$ in the language of forcing with respect to $j(\mathbb{P})$ such that if $G * H$ is (equivalently) $V$-generic or $M$-generic over $\mathbb{P} * \dot{\mathbb{Q}}, \Vdash_{j(\mathbb{P})}$ " $\tau$ extends every $j(\dot{q})$ for $\dot{q} \in \dot{H}$ ". In other words, $\tau$ is a term for a "master condition" for $\dot{\mathbb{Q}}$. Thus, if $\left\langle\dot{A}_{\alpha}: \alpha<\rho<\sigma\right\rangle$ enumerates in $V$ the canonical $\mathbb{P} * \dot{\mathbb{Q}}$ names of subsets of $\left(P_{\kappa}(\lambda)\right)^{V[G][H]}$, we can define in $M$ a sequence of $\mathbb{P} * \dot{\mathbb{Q}} * \dot{\mathbb{Q}}_{\kappa}^{1}$ names of elements of $\mathbb{R} * j(\dot{\mathbb{Q}})$, $\left\langle\dot{p}_{\alpha}: \alpha \leq \rho\right\rangle$, such that $\dot{p}_{0}$ is a term for $\langle 0, \tau\rangle$ (where 0 represents the trivial condition with respect to $\mathbb{R}), \Vdash_{\mathbb{P} * \dot{\mathbb{Q}} * \dot{\mathbb{Q}}_{\kappa}^{1}}$ " $\dot{p}_{\alpha+1}$ is a term for an Easton extension of $\dot{p}_{\alpha} \operatorname{deciding}$ ' $\langle j(\beta): \beta<\lambda\rangle \in j\left(\dot{A}_{\alpha}\right)$ '" , and for $\lambda \leq \rho$ a limit ordinal, $\Vdash_{\mathbb{P} * \dot{\mathbb{Q}}_{*} \dot{\mathbb{Q}}_{\kappa}^{1}}$ " $\dot{p}_{\lambda}$ is a term for an Easton extension of each member of the sequence $\left\langle\dot{p}_{\beta}: \beta<\lambda\right\rangle$ ". In $V[G][H]$, we may now define a set $\mathcal{U} \subseteq 2^{[\lambda]^{<\kappa}}$ by $X \in \mathcal{U}$ iff $X \subseteq P_{\kappa}(\lambda)$ and for some $\langle r, q\rangle \in G * H$ and some $q^{\prime} \in \mathbb{Q}_{\kappa}^{1}$ either the trivial condition (if $\mathbb{Q}_{\kappa}^{1}$ is trivial forcing) or of the form $\langle\emptyset, B\rangle$ (if $\mathbb{Q}_{\kappa}^{1}$ is Prikry forcing), in $M,\left\langle r, \dot{q}, \dot{q}^{\prime}, \dot{p}_{\rho}\right\rangle \Vdash "\langle j(\beta): \beta<\lambda\rangle \in j(\dot{X})$ " for some name $\dot{X}$ of $X$. As in [10, Lemma 2$], \mathcal{U}$ is a $\kappa$-additive, fine ultrafilter over $\left(P_{\kappa}(\lambda)\right)^{V[G][H]}$, i.e., $V[G][H] \vDash$ " $\kappa$ is $\lambda$ strongly compact". Since $\lambda$ was arbitrary, this completes the proof of Lemma 2.4 .

Lemma 2.5 $V^{\mathbb{P}} \vDash " N o$ cardinal $\delta<\kappa$ is strongly compact".

Proof: By the definition of $\mathbb{P}$ and the fact that for any supercompact cardinal $\kappa$, there are unboundedly in $\kappa$ many strong cardinals below $\kappa, V^{\mathbb{P}} \vDash$ "Unboundedly in $\kappa$ many cardinals $\delta<\kappa$ contain non-reflecting stationary sets of ordinals of cofinality $\omega "$. By [22, Theorem 4.8] and the succeeding remarks, $V^{\mathbb{P}} \vDash$ "No cardinal $\delta<\kappa$ is strongly compact". This completes the proof of Lemma 2.5.

Lemmas $2.1-2.5$ and the intervening remarks complete the proof of Theorem 1. 
By [11, Theorem 5], we know that if $\kappa$ is indestructibly supercompact and level by level equivalence between strong compactness and supercompactness holds, then no cardinal $\lambda>\kappa$ is $2^{\lambda}$

supercompact. However, the proof of [11, Theorem 5] does not rule out a positive answer to the question posed at the beginning of this paper. We therefore conclude by reiterating this question and asking, more generally, if it is possible to have a model containing many supercompact cardinals in which level by level equivalence between strong compactness and supercompactness holds and each supercompact cardinal has its supercompactness indestructible under the sorts of partial orderings we have just used.

\section{References}

[1] A. Apter, "Failures of GCH and the Level by Level Equivalence between Strong Compactness and Supercompactness", Mathematical Logic Quarterly 49, 2003, 587-597.

[2] A. Apter, "Failures of SCH and Level by Level Equivalence", Archive for Mathematical Logic 45, 2006, 831-838.

[3] A. Apter, "On the Non-Extendibility of Strongness and Supercompactness through Strong Compactness", Fundamenta Mathematicae 174, 2002, 87-96.

[4] A. Apter, "Patterns of Compact Cardinals", Annals of Pure and Applied Logic 89, 1997, $101-115$.

[5] A. Apter, "Supercompactness and Level by Level Equivalence are Compatible with Indestructibility for Strong Compactness", Archive for Mathematical Logic 46, 2007, 155-163.

[6] A. Apter, "Supercompactness and Measurable Limits of Strong Cardinals", Journal of Symbolic Logic 66, 2001, 629-639.

[7] A. Apter, "Supercompactness and Measurable Limits of Strong Cardinals II: Applications to Level by Level Equivalence", Mathematical Logic Quarterly 52, 2006, 457-463. 
[8] A. Apter, J. Cummings, "Identity Crises and Strong Compactness", Journal of Symbolic Logic 65, 2000, 1895-1910.

[9] A. Apter, J. Cummings, "Identity Crises and Strong Compactness II: Strong Cardinals", Archive for Mathematical Logic 40, 2001, 25-38.

[10] A. Apter, M. Gitik, "The Least Measurable can be Strongly Compact and Indestructible", Journal of Symbolic Logic 63, 1998, 1404-1412.

[11] A. Apter, J. D. Hamkins, "Indestructibility and the Level-by-Level Agreement between Strong Compactness and Supercompactness", Journal of Symbolic Logic 67, 2002, 820-840.

[12] A. Apter, S. Shelah, "On the Strong Equality between Supercompactness and Strong Compactness", Transactions of the American Mathematical Society 349, 1997, 103-128.

[13] M. Gitik, "Changing Cofinalities and the Nonstationary Ideal", Israel Journal of Mathematics $56,1986,280-314$.

[14] M. Gitik, S. Shelah, "On Certain Indestructibility of Strong Cardinals and a Question of Hajnal", Archive for Mathematical Logic 28, 1989, 35-42.

[15] J. D. Hamkins, Lifting and Extending Measures; Fragile Measurability, Doctoral Dissertation, University of California, Berkeley, 1994.

[16] J. D. Hamkins, "The Lottery Preparation", Annals of Pure and Applied Logic 101, 2000, 103-146.

[17] T. Jech, Set Theory: The Third Millennium Edition, Revised and Expanded, Springer-Verlag, Berlin and New York, 2003.

[18] J. Ketonen, "Strong Compactness and Other Cardinal Sins", Annals of Mathematical Logic $5,1972,47-76$.

[19] R. Laver, "Making the Supercompactness of $\kappa$ Indestructible under $\kappa$-Directed Closed Forcing", Israel Journal of Mathematics 29, 1978, 385-388. 
[20] A. Lévy, R. Solovay, "Measurable Cardinals and the Continuum Hypothesis", Israel Journal of Mathematics 5, 1967, 234-248.

[21] T. Menas, "On Strong Compactness and Supercompactness", Annals of Mathematical Logic 7, 1974, 327-359.

[22] R. Solovay, W. Reinhardt, A. Kanamori, "Strong Axioms of Infinity and Elementary Embeddings", Annals of Mathematical Logic 13, 1978, 73-116. 